\title{
A Study on Electrical and Thermal Properties of Polyimide/MWNT Nanocomposites
}

\author{
Soo-Jin Park, ${ }^{*}$ Sung-Won Chae, ${ }^{\dagger}$ John-Moon Rhee, ${ }^{\dagger}$ and Shin Jae Kang ${ }^{\star}$ \\ Dept. of Chemistry, Inha University, Incheon 402-751, Korea. ${ }^{*}$ E-mail: sjpark@inha.ac.kr \\ ${ }^{\dagger}$ Dept. of Polymer-Nano Science and Technology, Chonbuk National University, Jeonju 561-765, Korea \\ "Division of Mechanical Design Engineering, Chonbuk National University, Jeonju 561-765, Korea \\ Received June 11, 2009, Accepted June 22, 2010
}

\begin{abstract}
In this work, the electrical and thermal properties of polyimide/multi-walled carbon nanotube (MWNT) nanocomposites were investigated. The polyimide/MWNT nanocomposites contained from 0 to $2.0 \mathrm{wt} \%$ of MWNT. The electrical properties of the polyimide films were characterized by a specific resistance measurement. The thermal properties were evaluated using thermogravimetric analysis (TGA) and a differential scanning calorimeter (DSC). It was found that the thermal properties of the polyimide nanocomposites increased with increasing MWNT content and specific resistance as well. This result indicated that the crosslinking of polyimide/MWNT nanocomposites was enhanced by good distribution of the MWNT in the polyimide resins, resulting in the increase of the electrical and thermal properties of the nanocomposites.
\end{abstract}

Key Words: Polyimide, Multi-walled carbon nanotubes (MWNT), Chemical treatment, Thermal properties, Electrical properties

\section{Introduction}

Based on its unique chemical, thermal and mechanical characteristics, polyimide has been widely used in the fabrication of aircraft structures and microelectronic devices. However, due to its insulating nature, electrostatic charges can accumulate on the surfaces of polyimide. This causes local heating and, consequently, premature degradation of materials. ${ }^{1-3}$ This work describes MWNT reinforced polyimide nanocomposites with a level of electrical conductivity sufficient to permit electrostatic discharge, as well as mechanical and thermal properties superior to that of the base polymer.

Carbon nanotubes (CNTs) with a quasi-one-dimensional structure can be metallic or semiconducting, depending on their structural parameters. This feature has made CNTs central elements in composite materials for many electronic applications. CNTs also have high stiffness and tensile strength, which make them preferable for use in composites that require those mechanical properties. ${ }^{4} \mathrm{MWNT}$ have been used in composite materials to improve electrical properties while reinforcing the mechanical and thermal performance of the composites. ${ }^{5-8}$ Also, many efforts have been made to exploit the electrical, mechanical and thermal properties of polymer-CNTs composites by controlling the orientation of MWNT and polymer molecular chains. ${ }^{9-10}$ There are two common processing techniques for fabricating the composites. One is to mix MWNT with a resin matrix in the melting state to form composites. The other is to disperse MWNT into a polymer solution, cast the solution, and then remove the polymer using a solvent to obtain the composite. It is difficult to draw definite conclusions on electrical conductivity of polymer/MWNT nanocomposites from the literatures. This is because the reported level of MWNT loading for a percolation threshold varies widely, ranging from less than $1 \%$ to over $10 \%$. Two reasons are thought to account for the discrepancies: the MWNT bundles may not have been well dispersed in the polymer matrices, and experimental difficulties may have resulted in an erroneous detection at the onset of electric conduction. ${ }^{11}$

To pursue more perfect dispersion of MWNT in a polymer matrix, the MWNT used in this study were pre-treated in a solvent under mechanical stirring to form loose networks, and in situ polymerization of polyimide with fillers was adopted to fabricate the polyimide/MWNT composites. ${ }^{4}$ Park et al. ${ }^{12}$ prepared single-walled carbon nanotubes (SWNT)/polyimide composites by in situ polymerization, in which SWNT were dispersed homogeneously in the polyimide matrix. Qu et al. ${ }^{13}$ reported on the homogeneous dispersion of SWNT and MWNT into polyimide matrices for high quality nanocomposites. Zhu et $a l .{ }^{14}$ prepared Polyimide/MWNT nanocomposites via an efficient solution process, and the composites had a higher tensile strength with good electrical and dielectric properties. Yu et $a l .{ }^{15}$ developed polyimide/SWNT and in situ polymerization. In situ polymerization with dispersions of individual SWNT resulted in a composite film with good optical properties and enhanced mechanical, thermal and electrical properties. ${ }^{16}$

In this study, an in situ polymerization process of polyimide with MWNT is proposed for fabricating composite films in order to study their electrical properties. The mechanical properties and thermal stability of the fabricated composite films are also investigated.

\section{Experimental}

MWNT produced by chemical vapor deposition (CVD) were obtained from Iljin Co., Korea. The diameters of the MWNT were $10-20 \mathrm{~nm}$ and their lengths were $10-50 \mu \mathrm{m}$. The matrix used in this study was a polyimide (Sang-A FLONTEC Co., Korea), with a viscosity of about $5,000 \mathrm{cps}$ at $25^{\circ} \mathrm{C}$. The precursor of polyimide was prepared by reacting 4,4'-oxydianiline (ODA, Aldrich) with 3,3',4,4'-benzophenone tetracarbox- 


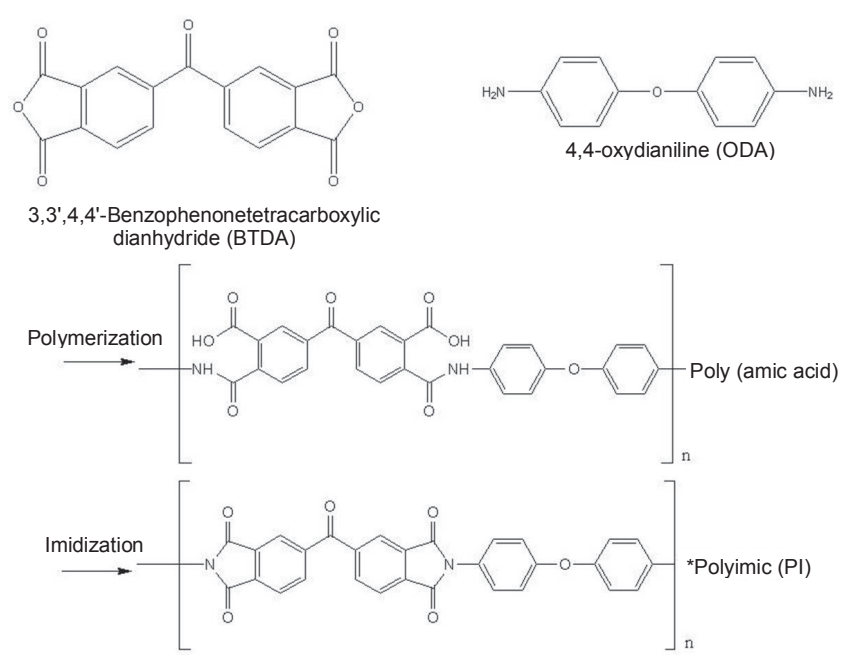

Figure 1. Polymerization process of polyimide.

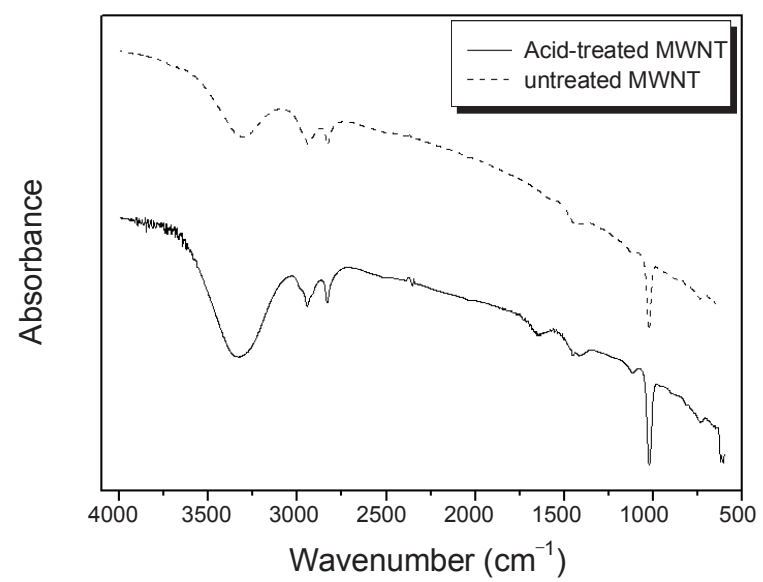

Figure 2. FT-IR spectra of untreated MWNT and acid-treated MWNT.



Figure 3. XPS survey scan spectra of untreated MWNT and acid-treated MWNT.

ylic dianhydride (BTDA, Aldrich) in a vacuum oven for $24 \mathrm{~h}$. The solvent used was $n$-methyl-1-pyrrolidinone (NMP, Aldrich). The monomers, ODA and with BTDA were added in equimolar amounts to the NMP solution. After stirring the solutions for $24 \mathrm{~h}$ in nitrogen gas at ambient temperature, polyamic acid was obtained. The concentration of the solution was 15 - $20 \mathrm{wt} \%$ oli- gomer against NMP. One gram of the crude MWNT was purified using $150 \mathrm{~mL}$ with $20 \mathrm{wt} \%$ of dilute nitric acid for $24 \mathrm{~h}$ at $40^{\circ} \mathrm{C}$ in order to remove the unwanted carbon and metal catalysts. Then, the purified MWNT were chemically modified using a mixed acid: $0.5 \mathrm{~g}$ of the MWNT was suspended in $80 \mathrm{~mL}$ of a $3: 1(\mathrm{v} / \mathrm{v} \%)$ mixture of concentrated sulfur acid (98\%) and nitric acid $(67 \%)$ and stirred for $5 \mathrm{~h}$ at room temperature. ${ }^{17}$ The resultant acid-treated MWNT were then washed using distilled water with a $\mathrm{pH}$ of 7 , and dried at $80^{\circ} \mathrm{C}$ in a vacuum oven. Next, the modified MWNT of $0.5,1.0,1.5$ and $2.0 \mathrm{wt} \%$ were manually suspended in polyamic acid and then stirred for $4 \mathrm{~h}$ using a mechanical stirrer. Thereafter, the blends were degassed for $30 \mathrm{~min}$ in a vacuum oven. The polyamic acid solutions with MWNT were cast onto clean, dry plate glass using an applicator (Sheen Instrument, S220403), and then dried to a tack-free state at $60^{\circ} \mathrm{C}$ for $24 \mathrm{~h}$. The thickness of the films was $50 \mu \mathrm{m}$. Then, the composite polyamic acid films were cured for 75 min at $50{ }^{\circ} \mathrm{C}, 75 \mathrm{~min}$ at $150{ }^{\circ} \mathrm{C}, 75 \mathrm{~min}$ at $250{ }^{\circ} \mathrm{C}$, and $5 \mathrm{~min}$ at $280^{\circ} \mathrm{C}$. The heating rate was $2^{\circ} \mathrm{C} / \mathrm{min}$, and the cooling rate was $1{ }^{\circ} \mathrm{C} / \mathrm{min}$. The polymerization process of polyimide is illustrated in Figure 1.

Fourier transform infrared (FT-IR) spectroscopic measurements were performed to identify the structure of the synthesized samples using a FT-IR 610 (Jasco, Japan) equipped with attenuated total reflection. X-ray photoelectron spectroscopy (XPS) was studied using an ESCALAB 200R (West Sussex, U.K.). Thermogravimetric analysis (TGA) was performed with a TGA 2050(TA Instruments, USA) at a $10{ }^{\circ} \mathrm{C} / \mathrm{min}$ heating rate under nitrogen purge. Differential scanning calorimetry (DSC) studies were carried out using a DSC 2910 (TA Instruments, USA). Electrical resistance measurement was carried out using a Mitsubishi Chemical Corp. LORESTA-GP (MCP-T610).

\section{Results and Discussion}

The FT-IR spectra of untreated and acid-treated MWNT are shown in Figure 2. Compared with untreated MWNT, the acidtreated MWNT possess the polar groups. That is, the acid-treated MWNT have four peaks near at $3450 \mathrm{~cm}^{-1}$ (-OH stretching vibration in -C-OH group), $2980 \mathrm{~cm}^{-1}$ (C-C group), $1680 \mathrm{~cm}^{-1}$ ( $-\mathrm{C}=\mathrm{O}$ group), and $1075 \mathrm{~cm}^{-1}$ (N-H stretching vibration) regions. ${ }^{18-19}$ Compared with the untreated MWNT, it is confirmed that the surfaces of the modified MWNT possess the polar groups of carboxyl and hydroxyl groups.

Figure 3 shows the XPS spectra of the MWNT before and after acid treatments. It was found that the chemical composition was significantly changed after the treatments. The peaks at 294.8 and $532.2 \mathrm{eV}$ are attributed to $\mathrm{C}$ and $\mathrm{O}$, respectively. As expected, the oxygen peak intensity is increased after the acid treatment. The content of elements on the surfaces of the MWNT is calculated by the area of each element. The $\mathrm{O}_{1 \mathrm{~s}} / \mathrm{C}_{1 \mathrm{~s}}$ in untreated MWNT is 0.014, while that of the acid-treated MWNT is 0.065 . According to the results of XPS measurement, it can be surmised that functional groups like the carboxyl group, carbonyl group and hydroxyl group were further formed by acid-treatment.

In order to study the influence of acid treatments on the integrity of MWNT, the morphology of untreated MWNT and 


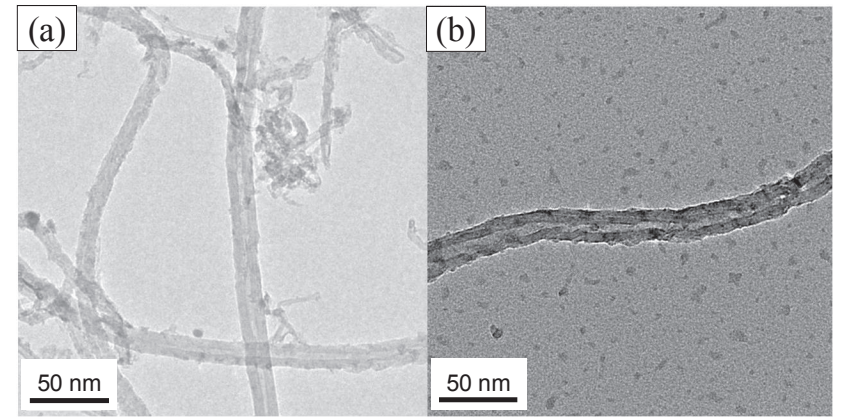

Figure 4. TEM images of untreated (a) and acid-treated MWNT (b).

Table 1. $\mathrm{C}_{1 \mathrm{~s}}$ and $\mathrm{O}_{1 \mathrm{~s}}$ values fot the untreated and acid-treated MWNT by XPS measurement.

\begin{tabular}{cccc}
\hline Samples & $\mathrm{C}_{1 \mathrm{~s}}$ at $(\%)$ & $\mathrm{O}_{1 \mathrm{~s}}$ at $(\%)$ & $\mathrm{O}_{1 \mathrm{~s}} / \mathrm{C}_{1 \mathrm{~s}}$ ratio \\
\hline Untreated MWNT & 98.65 & 1.35 & 0.014 \\
Acid-treated MWNT & 93.94 & 6.06 & 0.065 \\
\hline
\end{tabular}

Table 2. Thermal stability of chemically modified polyimide/MWNT nanocomposites.

\begin{tabular}{cccccc}
\hline $\begin{array}{c}\text { MWNT } \\
\text { Content }(\mathrm{wt} \%)\end{array}$ & IDT $\left({ }^{\circ} \mathrm{C}\right)$ & $\mathrm{T}_{\max }\left({ }^{\circ} \mathrm{C}\right)$ & $\mathrm{A}^{*} \mathrm{~K}^{*}$ & $\begin{array}{c}\mathrm{IPDT} \\
\left({ }^{\circ} \mathrm{C}\right)\end{array}$ & $\begin{array}{c}\mathrm{Et} \\
(\mathrm{kJ} / \mathrm{mol})\end{array}$ \\
\hline 0.5 & 533 & 605 & 3.09 & 2410 & 35 \\
1.0 & 550 & 608 & 3.33 & 2586 & 34 \\
1.5 & 549 & 607 & 3.26 & 2537 & 31 \\
2.0 & 555 & 606 & 2.82 & 2202 & 58 \\
\hline
\end{tabular}

acid-treated MWNT were observed using TEM. As shown in Figure 4, there is no apparent change on the diameter and wall thickness, demonstrating that acid treatments did not cause apparent detrimental effects on the MWNT structure.

Table 2 displays the thermal stability parameter of the polyimide/MWNT in an environment of nitrogen. The initial decomposition temperature (IDT) of these nanocomposites seems to increase with increased MWNT content. The dispersion of MWNT in the polyimide matrix enhances the thermal resistance of polyimide/MWNT because the MWNT bond chemically or physically interact with the polyimide matrix. With increasing MWNT loading from 0.5 to $2.0 \mathrm{wt} \%$, the IDT of the polyimide/ MWNT nanocomposites increased from 533 to $555^{\circ} \mathrm{C}$. The increase in the IDT of the polyimide/MWNT nanocomposites depends on the degree of dispersion of the MWNT, showing the efficient dispersion of MWNT in the polyimide matrix. The integral procedural decomposition temperature (IPDT), IDT and temperature of maximum rate of weight loss $\left(\mathrm{T}_{\max }\right)$ increased with higher MWNT contents.

The glass transition temperature $\left(\mathrm{T}_{\mathrm{g}}\right)$ of the polymer matrix depends on the free volume of the polymer, which is related to the affinity between the filler and the polymer matrix. The acidtreated MWNT have a better affinity for the polymer matrix than the untreated MWNT. The acid-treated MWNT may form hydrogen bonds with the $\mathrm{C}=\mathrm{O}$ groups of the polyimide molecules. The $\mathrm{T}_{\mathrm{g}}$ values of the polyimide/MWNT nanocomposites were investigated by DSC, and illustrated in Figure 6. The acid-

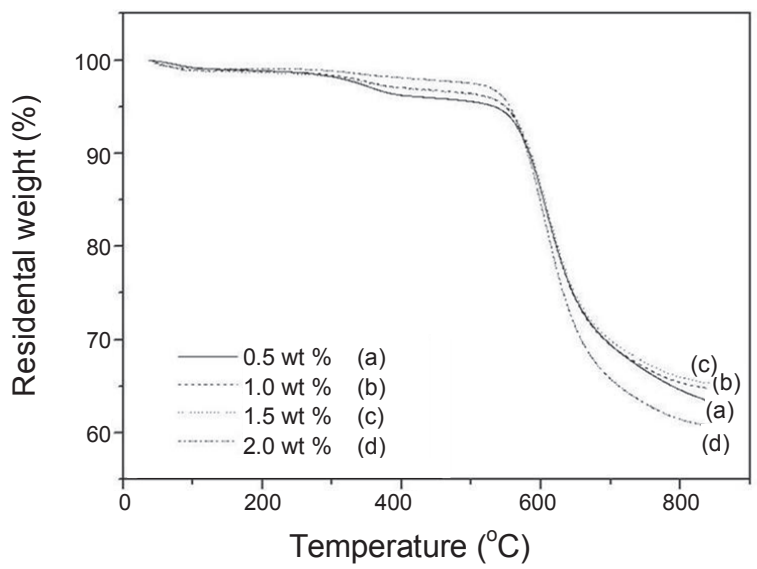

Figure 5. TGA diagrams of various polyimide/MWNT nanocomposites.



Figure 6. DSC diagrams of various polyimide/MWNT nanocomposites.

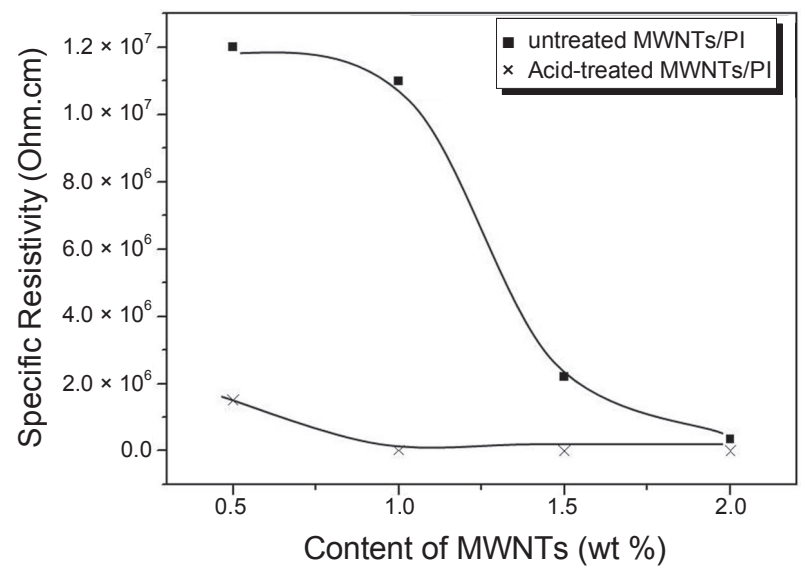

Figure 7. Dependence of electrical resistivity on the MWNT content for polyimide/MWNT nanocomposites.

treated MWNT caused increased $\mathrm{T}_{\mathrm{g}}$ of the polyimide composite. The $\mathrm{T}_{\mathrm{g}}$ of the $2.0 \mathrm{wt} \%$ acid-treated polyimide/MWNT was $282.7{ }^{\circ} \mathrm{C}$. As a result, the $\mathrm{T}_{\mathrm{g}}$ increased gradually as the content of acid-treated MWNT increased, because the free volume of the composites decreased to the extent of $2.0 \mathrm{wt} \%$ MWNT. 
The electrical resistivity of the polyimide/MWNT nanocomposites as a function of MWNT is shown in Figure 7. The electrical resistivity of the polyimide/MWNT nanocomposites decreased as MWNT content increased. The abrupt decrease in the electrical resistivity for MWNT up to $1.0 \mathrm{wt} \%$ suggests that the percolation threshold in the electrical resistivity of the nanocomposites is around $1.0 \mathrm{wt} \%$ content. When the MWNT concentration was increased further, the electrical resistivity decreased slowly, and the composites seemed to reach a saturation point beyond which resistivity no longer decreased. The electrical resistivity of the acid-treated polyimide/MWNT composites was lower than that the untreated MWNT composites at identical MWNT content. As a result, the electrical current of the composite filled with the well-dispersed MWNT was higher than that of the composite filled with the poorly dispersed MWNT. It was easier for the well-dispersed MWNT to form electrical paths due to their relatively homogeneous dispersion of nanoparticles than it was for the poorly dispersed MWNT. This is due to the increased number of defects in the lattice structure of the carbon-carbon bonds formed on the nanotube surfaces, as well as to the decreased aspect ratio of the tubes after acid treatment.

\section{Conclusions}

Polyimide/MWNT nanocomposites were prepared by in situ polymerization. Compared with the untreated MWNT, the surfaces of the modified MWNT possessed a higher portion of the polar groups of carboxyl and hydroxyl groups. This result played an important role in improving the degree of adhesion at interfaces between the polyimide and the MWNT. The glass transition temperature and thermal stability were increased when acid-treated MWNT were used in polyimide/MWNT nanocomposites. The hydrogen atoms at the $\mathrm{COOH}$ groups of acidtreated MWNT may form hydrogen bonds with the $\mathrm{C}=\mathrm{O}$ groups of the polyimide molecules. The electrical resistivity of the polyimide/MWNT composites decreased with an increase in the MWNT content. The specific resistance of the polyimide/
MWNT nanocomposites containing 0.5 - $1.0 \mathrm{wt} \%$ MWNT was abruptly decreased, due to the formation of the conduction network by highly dispersed MWNT.

Acknowledgments. This work was supported by the Materials Source Technology Development Program of the Ministry of Knowledge Economy, Korea.

\section{References}

1. Ajayan, P. M.; Ebbesen, T. W.; Ichihashi, T.; Iijima, S.; Tanigaki, K.; Hiura, H. Nature 1993, 362, 522.

2. Breuer, O.; Sundararaj, U. Polym. Compos. 2004, 25, 630.

3. Jiang, X.; Bin, Y.; Matsuo, M. Polymer 2005, 46, 7418.

4. Imai, Y.; Fueki, T.; Inoue, T.; Kakimoto, M. J. Polym. Sci. Technol. 1998, 61, 1899.

5. Popov, V. N. Mater. Sci. Eng. 2004, 43, 61.

6. Potschke, P.; Fornes, T. D.; Paul, D. R. Polymer 2002, 43, 3247.

7. Zhu, B. K.; Xie, S. H.; Xu, Z. K.; Xu, Y. Y. Compos. Sci. Technol. 2006, 66, 551 .

8. Cadek, M.; Coleman, J. N.; Barron, V.; Hedicke, K.; Blau, W. J. Appl. Phys. Lett. 2002, 81, 5123.

9. Bin, Y.; Kitanaka, M.; Zhu, D.; Matsuo, M. Macromolcules 2003, 36, 6213.

10. Yuen, S. M.; Ma, C. M.; Lin, Y. Y.; Kuan, H. C. Compos. Sci. Technol. 2007, 67, 2566.

11. Oumaies, Z.; Park, C.; Wise, K. E.; Siochi, E. J.; Harrison, J. S. Compos. Sci. Technol. 2003, 63, 1637.

12. Park, C.; Ounaies, Z.; Watson, K. A.; Crooks, R. E.; Smith, J. E.; Lowther, S. E. Chem. Phys. Lett. 2002, 364, 304.

13. Qu, L.; Lin, Y.; Hill, D. E.; Zhou, B.; Wang, W.; Sun, X. Macromolecules 2004, 37, 6056.

14. Zhu, B. K.; Xie, S. H.; Xu, Z. K.; Xu, Y. Y. Compos. Sci. Technol. 2006, 66, 549 .

15. Yu, A.; Hu, H.; Bekyarova, E.; Itkis, M. E.; Gao, J.; Zhao, B. Compos. Sci. Technol. 2006, 66, 1188.

16. Delozier, D. M.; Watson, K. A.; Smith, J. G.; Connell, J. W. Compos. Sci. Technol. 2005, 65, 753 .

17. Park, S. J.; Seo, M. K. Chem. Phys. Lett. 2004, 395, 44.

18. Endo, M.; Takeucho, K.; Hiraoka, T.; Furuta, T.; Kasai, T.; Sun, X. J. Phys. Chem. Solid 1997, 58, 1709.

19. Park, S. J.; Lee, E. J.; Lee, J. R.; Won, H. Y.; Moon, D. K. Polymer (Korea) 2007, 31, 120. 